\title{
What we know about surgical therapy in early- stage non-small-cell lung cancer: a guide for the medical oncologist
}

This article was published in the following Dove Press journal:

Cancer Management and Research

6 July 2017

Number of times this article has been viewed

\author{
Sassine Ghanem' \\ Sandy El Bitar' \\ Sami Hossri' \\ Chanudi Weerasinghe ${ }^{2}$ \\ Jean Paul Atallah ${ }^{2}$ \\ 'Department of Internal Medicine, \\ ${ }^{2}$ Department of Hematology and \\ Oncology, Staten Island University \\ Hospital - Northwell Health, New \\ York, NY, USA
}

\begin{abstract}
Lung cancer remains the leading cause of death in cancer patients. The gold standard for the treatment of early-stage non-small-cell lung cancer is lobectomy with mediastinal lymphnode dissection or systematic lymph-node sampling. The evidence behind this recommendation is based on the sole randomized controlled trial conducted to date, done by the Lung Cancer Study Group and published in 1995, which found a superiority for lobectomy over sublobar resection with regard to local recurrence rate and improved survival. The population studied at that time were medically fit patients at low risk for surgery with a stage IA non-small-cell lung carcinoma, ie, a solitary tumor less than $3 \mathrm{~cm}$ in size. In practice, however, thoracic surgeons have continued to push the limit of a more conservative surgical resection in this patient population. Since then, several retrospective studies have attempted to identify the ideal population to benefit from sublobar resection without it affecting survival or local recurrence. Several variables have been studied, including tumor size, patient age, surgical approach, histological and radiological properties, and optimal surgical resection margin, as well as promising prognostic biomarkers. In this review, we summarize the data available in the literature regarding the surgical approach to patients with stage IA non-small-cell lung cancer studying all the aforementioned variables. Keywords: segmentectomy, wedge resection, lung cancer, lobectomy, non-small-cell lung cancer, sublobar resection
\end{abstract}

\section{Introduction}

Despite all advances in screening and therapy, lung cancer remains the leading cause of cancer-related death in the US, with an estimated 224,390 new cases and 158,080 deaths annually. ${ }^{1}$ According to current guidelines, staging of lung cancer dictates the treatment plan. As per the seventh edition of TNM staging, early-stage lung cancer, specifically stage IA non-small-cell lung cancer (NSCLC), is defined as a tumor size of less than $3 \mathrm{~cm}$ surrounded by lung or visceral pleura without invasion more proximal than the lobar bronchus. ${ }^{2}$ The gold standard for the treatment of stage IA NSCLC has been lobectomy with mediastinal lymph-node dissection or systematic lymph-node sampling. ${ }^{3}$ The bulk of the evidence behind this recommendation is based on a single randomized controlled trial done by the Lung Cancer Study Group (LCSG) and published in $1995 .{ }^{4}$ The aim of this study was to try and prove noninferiority of sublobar resections, such as segmentectomy and wedge resection, when chosen electively in medically fit patients. Segmentectomy is a more technically challenging anatomic resection, while wedge resection is a nonanatomic resection where the pulmonary lesion is removed surrounded by a margin of normal lung parenchyma.
Correspondence: Sassine Ghanem Staten Island University Hospital, Northwell Health, 475 Seaview Ave, Staten Island, New York, NY 10305, USA Tel + I 3476855502

Email sghanem@northwell.edu 
However, the LCSG trial showed a threefold increase in local recurrence and a slightly significant decrease in overall survival (OS) following sublobar resection. It is important to note that no other randomized controlled study studying this subject has been conducted since, and the remainder of the evidence we have now stems mainly from retrospective analysis.

While lobectomy was standardized as the surgical treatment for stage IA NSCLC and remained so for the following 20 years, it would not be erroneous to assume that the population studied at the time the LCSG study completed enrollment in 1988 was different from the early-stage lung cancer population of today. This was prior to the introduction of positron-emission tomography with fludeoxyglucose, as well as the improvement in resolution of computed tomography (CT) scanners, which allow for better staging of the disease, better assessment of lymph-node involvement, and diagnosis of lung cancer at an earlier stage. Precise identification of minor changes in the density within ground-glass nodules and a better understanding of histological tumor biology introduced subgroups with indolent behavior and more favorable outcomes. In addition, the US Preventive Services Task Force since 2004 has integrated low-dose CT in adults aged 55-80 years who have a 30 pack-year smoking history and currently smoke or have quit within the past 15 years as part of their screening guidelines. Modeling studies conducted by the Cancer Intervention and Surveillance Modeling Network investigators for the Preventive Services Task Force suggest that implementing such a screening protocol would result in approximately $50 \%$ of lung cancer cases being detected at an early stage. ${ }^{5}$ Simultaneously, advances in surgical technique, as well as the use of video-assisted thoracoscopic surgery, which allow for better tolerated resections, have brought older and higher-risk populations into consideration for anatomic resection. In this review, we discuss recent data regarding the options available for the treatment of stage IA NSCLC, as well as the evidence guiding the choices on which patient population would benefit most from each technique.

\section{Surgical options}

The LCSG trial was fueled by a trend toward using sublobar resection in patients with stage IA NSCLC who are at low risk for surgery. To refer to these patients, we will be using the term "intentional" sublobar resection, as opposed to a "compromised" sublobar resection, where the elevated surgical risk or a low pulmonary reserve obliges the surgeon to opt for a sublobar resection. The LCSG trial analyzed data on 247 patients that were randomized to either lobectomy or sublobar resection. In patients undergoing sublobar resection, tripling of the local recurrence rate ( $P=0.008$, two-sided) was observed. The overall death rate increased by $30 \%$ and the cancer-related death rate increased by $50 \%$ in the sublobar resection group ( $P=0.094$, one-sided), which was significant. When the 12- to 18-month pulmonary function tests were compared with the baseline pulmonary function tests, the changes in both groups were similar and showed no statistically significant difference between the two methods. ${ }^{4}$ While the conclusion drawn from this study is that lobectomy should remain the standard of care for stage IA NSCLC, several limitations of this study should be pointed out.

The one-sided $P$-value used to test for significance was set at 0.1 , and thus the survival-rate difference was only barely significant and exceeded the usual cutoff value of 0.05 . The investigators in this study justified taking a higher $P$-value with the fact that this was an equivalence study, where higher $P$-values are used to avoid the more dangerous false-negative conclusion. Wedge resections and segmentectomies were categorized under the same group. Approximately 33\% of the patients in the sublobar resection group underwent wedge resection, which is not an anatomical dissection, and critics argue that it can be associated with a higher recurrence rate and thus might have skewed the results. CT scans were not performed on a routine basis to stage patients accurately. Finally, only $66 \%$ of patients were compliant with the 1 -year assessment of pulmonary function testing. Several surgeons remained skeptical, and subsequent retrospective analyses were performed attempting to validate or refute the findings of the LCSG trial, as well as to explore various outcomes according to tumor size, histology, patient characteristics, and surgical techniques used.

\section{Survival and recurrence rate}

Multiple retrospective series have studied survival- and recurrence-rate differences between patients treated with lobectomy versus sublobar resections and demonstrated conflicting results. Landrenau et al analyzed 219 consecutive patients with pathologic stage I (T1, N0, MO) that underwent either lobectomy or wedge resection (open or thoracoscopic approach), and found no difference in postoperative mortality between the different groups, with a $P$-value of 0.2 . Postoperative length of stay was shorter in the wedge-resection groups, with an average of 7.7 days using the open approach and 6.3 days using the video-assisted thoracoscopic surgery (VATS) approach vs 10.6 days for the lobectomy group, with a $P$-value of 0.0002 . Local recurrence rate was higher in the wedge-resection groups, with $24 \%$ and $16 \%$ using 
the open and VATS approach, respectively, versus $9 \%$ in the lobectomy group, but this result was nonsignificant, with a $P$-value of 0.07 . Although the patient population undergoing wedge resection included compromised patients that were generally older and with poorer pulmonary function, the Kaplan-Meier survival proportions for all causes appeared nearly identical at 1 year, and over the entire 5-year study period did not differ significantly between the wedge-resection groups (open or VATS approach) and the lobectomy group $(P=0.056)$. Non-cancer-related deaths, however, were higher in the wedge-resection groups than in the lobectomy group $(P=0.014)$. This difference was specifically higher in the open wedge-resection group compared to the lobectomy group, with a $P$-value of $0.005 .^{6}$

Khullar et al utilized the National Cancer Database to study retrospectively (after propensity-score matching) three groups of 987 patients with early NSCLC undergoing segmentectomy, wedge resection, or lobectomy. They found that 30 -day mortality was $1.6 \%$ for lobectomy, $1.51 \%$ for wedge resection, and $1.55 \%$ for segmentectomy, without any statistically significant difference $(P=0.868)$. Median OS for lobectomy, segmentectomy, and wedge resection was 99.5 (95\% CI 96.8-105), 74 (95\% CI 65.7-87.4), and 67.9 (95\% CI 63.6-71.3) months, respectively. Segmentectomy and wedge resection were thus associated with worse OS, with an HR of 1.7 (95\% CI 1.29-2.26, $P<0.001)$ and $1.45(95 \%$ CI $1.1-1.91, P=0.009)$, respectively. ${ }^{7}$

A systematic review and meta-analysis was then performed by $\mathrm{Cao}$ et al to try and reconcile the conflicting results from previous studies, including the aforementioned. A total of 54 studies from six online databases were selected and classified into three analysis groups: sublobar resection vs lobectomy, wedge resection vs lobectomy, and segmentectomy vs lobectomy. Inside each analysis group, the studies were also divided according to their patient selection criteria into "intentional", "compromised", and "not specified", then analyzed separately. Overall and disease-free survival were the outcomes studied. In the sublobar resection versus lobectomy group, comparative data demonstrated no significant difference in OS in the intentional group (HR $0.85,95 \%$ CI $0.46-1.57 ; P=0.6$ ), but significantly worse outcomes for sublobar resections in the compromised group (HR 1.41, 95\% CI 1.2-1.66; $P<0.0001$ ), as well as the unspecified group (HR $1.4,95 \%$ CI $1.32-1.48 ; P<0.00001)$. There were no studies included that looked at disease-free survival where patients were selected with intentional criteria. However, disease-free survival was inferior in both the compromised group (HR $1.48,95 \%$ CI $1.1-1.99 ; P=0.01)$ and the unspecified group
(HR 1.46, 95\% CI 1.07-1.99; $P=0.02$ ). In the segmentectomy versus lobectomy group, comparative data demonstrated no significant difference in OS in the intentional group (HR $0.94,95 \%$ CI $0.52-1.68 ; P=0.83$ ) or the unspecified group (HR $1.05,95 \%$ CI $0.83-1.32 ; P=0.7$ ), but significantly worse outcomes for segmentectomy in the compromised group (HR 1.56, 95\% CI 1.05-2.32; $P=0.03$ ). There was no significant difference in disease-free survival in the intentional (HR 0.94, 95\% CI 0.52-1.68; $P=0.37$ ), compromised (HR $1.25,95 \%$ CI $0.86-1.81 ; P=0.25$ ), or unspecified (HR 1.29, $95 \%$ CI $0.64-2.58 ; P=0.48)$ groups. In the wedge resection versus lobectomy group, comparative data demonstrated no significant difference in OS in the intentional group (HR $0.75,95 \%$ CI $0.11-5.11 ; P=0.77)$, but a significantly worse outcomes for wedge resection in the compromised group (HR $1.8,95 \%$ CI $1.26-2.57 ; P=0.001)$, as well as the unspecified group (HR 1.52, 95\% CI 1.12-2.06; $P=0.007$ ). There were no studies included that looked at disease-free survival where patients were selected with unspecified criteria. Disease-free survival was similar in the intentional group (HR 0.54, 95\% CI $0.14-2.1 ; P=0.38)$, but worse in the compromised group (HR 2.01, 95\% CI 1.3-3.09; $P=0.002$ ). The main conclusion to be drawn from this meta-analysis is that sublobar resection, specifically segmentectomy, when chosen in the appropriate intentionally selected patient, has similar overall and disease-free survival. To identify those patients who were potentially suitable for sublobar resections, the selection process of individual studies involving the intentionally selected patients was extracted from the eleven identified comparative studies. The common criteria found were tumor size $<2 \mathrm{~cm}$, peripheral location with a margin of at least $2-3 \mathrm{~cm}$, and a high proportion of ground-glass opacity (GGO) demonstrated on high-resolution CT (HRCT). ${ }^{8}$

\section{Tumor size}

The cutoff for tumor size used in the LCSG trial was $3 \mathrm{~cm} .{ }^{4}$ Several researchers have attempted to study whether smaller tumors might have shown oncologic equivalence between lobectomy and sublobar resection. Okada et $\mathrm{al}^{9}$ reviewed the records of 1,272 consecutive patients who underwent complete resection for NSCLC. These patients were divided using tumors of $10 \mathrm{~mm}$ or less, $10-20 \mathrm{~mm}, 20-30 \mathrm{~mm}$, and greater than $30 \mathrm{~mm}$ in diameter. The 5-year overall and cancer-specific survival were the outcomes studied after lobectomy, segmentectomy, or wedge resection. Overall 5-year survival for patients with pathologic stage I disease in these four groups was $86 \%, 83.8 \%, 75.3 \%$, and $67 \%$, respectively. Cancer-specific 5-year survival for patients with 
pathologic stage I disease in these four groups was $100 \%$, $92.6 \%, 84.1 \%$, and $76.4 \%$, respectively. It is important to note that with all surgical procedures combined, 5-year overall and cancer-specific survival for NSCLC tumors 10 $20 \mathrm{~mm}$ and $20-30 \mathrm{~mm}$ was statistically significant, with $P=0.097$ and $P=0.006$, respectively. On the other hand, 5 year overall and cancer-specific survival for NSCLC tumors 20-30 $\mathrm{mm}$ and above $30 \mathrm{~mm}$ was similar, with $P=0.2199$ and $P=0.1101$, respectively. Okada et al then divided patients with pathologic stage I NSCLC into three groups of tumor size $20 \mathrm{~mm}$ and less, $20-30 \mathrm{~mm}$, and greater than $30 \mathrm{~mm}$ in diameter and analyzed cancer-specific survival according to the operative procedure used. After lobectomy, the 5-year cancer-specific survival rate for tumors $20 \mathrm{~mm}$ and less, 20-30 mm, and greater than $30 \mathrm{~mm}$ in diameter was $96.7 \%$, $87.4 \%$, and $81.3 \%$, respectively. After segmentectomy, the 5-year cancer-specific survival rate for tumors $20 \mathrm{~mm}$ and less, 20-30 mm, and greater than $30 \mathrm{~mm}$ in diameter was $92.4 \%, 84.6 \%$, and $62.9 \%$ respectively. After wedge resection, the 5-year cancer-specific survival rate for tumors 20 $\mathrm{mm}$ and less, $20-30 \mathrm{~mm}$, and greater than $30 \mathrm{~mm}$ in diameter was $85.7 \%, 39.4 \%$, and 0 , respectively. ${ }^{9}$ It is based on this study, among others with similar findings, that the seventh edition of the TNM staging now subdivides T1 into T1a and T1b, with $20 \mathrm{~mm}$ as the cutoff value. ${ }^{2}$

Bao et al conducted a meta-analysis of 22 observational studies and compared OS and cancer-specific survival with lobectomy vs segmentectomy after dividing patients into stage I, stage IA, stage IA with tumors greater than 2 but less than $3 \mathrm{~cm}$, and stage IA with tumors less than or equal to $2 \mathrm{~cm}$. They found that there was no difference in overall/cancer-specific survival for patients with stage IA and tumors less than or equal to $2 \mathrm{~cm}$ (HR 1.05, 95\% CI 0.89$1.24 ; P=0.055)$. However, for patients classified as stage $\mathrm{I}$, stage IA, and stage IA with tumor size between 2 and $3 \mathrm{~cm}$, there existed a statistically significant difference in overall/ cancer-specific survival, with a $P$-value of $0.011,0.002$, and 0.001 , respectively. ${ }^{10}$

\section{Age}

Age is an important factor in deciding on preoperative risk, and plays a role in prognosis following lobectomy or sublobar resection. In 2005, Mery et al studied the effect of age and type of surgery on long-term survival in patients with early-stage NSCLC. They analyzed the data of a total of 14,555 patients with stage I or II primary NSCLC that had been registered in the Surveillance, Epidemiology, and End Results (SEER) database from 1992 to 1997. They divided the patients into three age-groups: age less than 65 years, 65-74 years, and greater than or equal to 75 years. As expected, survival decreased with age, and median survival times were 71, 47, and 28 months, respectively, for each age-group. In younger patients, a survival benefit was noted for those undergoing lobectomy compared to patients with limited resections. This was not true of elderly patients in this study, and the age at which this benefit was lost was calculated to be 71. OS for patients of age less than or equal to 71 years was higher in patients undergoing lobectomy vs limited resection, with $P=0.004$. On the other hand, OS for patients of age greater than or equal to 72 years was similar in patients undergoing lobectomy vs limited resection, with a $P=0.27$. $^{11}$

A more recent trial querying the SEER database was done by Razi et al, and studied 1,640 patients aged older than or equal to 75 years who had been diagnosed with stage IA NSCLC from 1998 to 2007. Patients were then divided into three groups based on type of surgery performed: wedge resection, segmentectomy, and lobectomy. Cox regression multivariate analysis of 5-year cancer specific survival for stage IA (T1a/b) NSCLC patients showed similar survival for wedge resection and segmentectomy, with an HR of 0.797 and $P=0.25$. Lobectomy, however, showed a superior survival with a $\mathrm{HR}$ of 0.764 and $P=0.032$. A similar analysis limited only to patients with T1a NSCLC showed similar survival between wedge resection and segmentectomy, with an HR of 1.009 and $P=0.972$, or lobectomy, with an HR of 0.98 and $P=0.908 .{ }^{12}$

\section{Segmentectomy vs wedge resection}

Sublobar resection is further divided into segmentectomy and wedge resection. Segmentectomy is a resection that limits itself to the anatomical borders of the segment in which the tumor lies. A wedge resection is a nonanatomical resection that simply removes the tumor with a margin of normal lung parenchyma surrounding it. There are two main dilemmas that the surgeon is confronted with while performing a sublobar resection. The first dilemma consists of the distance between the tumor and operating margin that is considered adequate. Local recurrence rates are inversely correlated with an increase in margin distance. El-Sherif et al reviewed 81 NSCLC patients who underwent sublobar resection. In their study, six of 41 patients $(14.6 \%)$ developed local recurrence in the group with a margin $<1 \mathrm{~cm}$ versus three of 40 patients $(7.5 \%)$ in the group with a margin $\geq 1 \mathrm{~cm}(P=0.04)$. Of note, in patients that were operated on with segmentectomy, 19 (73\%) had a margin of $\geq 1 \mathrm{~cm}$ and seven (27\%) had a margin 
of only $<1 \mathrm{~cm} .{ }^{13}$ Based on these and other data, the current recommendation by the National Comprehensive Cancer Network is that sublobar resection should achieve a parenchymal resection margin $\geq 2 \mathrm{~cm}$ or greater than or equal to the size of the nodule. ${ }^{3}$

The second dilemma consists of the extent of lymph-node dissection that should be performed. The nonanatomical nature of wedge resection further complicates the decision on which lymph-node stations should be resected. Lymph-node dissection is essential, since small tumors do not correlate well with lymph-node negativity. The incidence of N1 and $\mathrm{N} 2$ nodal involvement in peripheral clinical stage I NSCLC $\leq 2 \mathrm{~cm}$ in diameter was $5.3 \%$ and $6.6 \%$, respectively. In tumors $<1 \mathrm{~cm}$ in diameter, N2 disease was still present in 3.8\% of them. ${ }^{14}$ In the meta-analysis done by Cao et al, it was shown that hilar and mediastinal lymph-node sampling or dissection was more likely to accompany segmentectomies than wedge resections. ${ }^{8}$ Larger margins and a higher probability for lymph-node dissection could explain the superiority of segmentectomies.

\section{Histology, radiology, and pathological invasiveness}

A better understanding of lung cancer histology and specifically adenocarcinoma has shown that adenocarcinoma of the lung is not a uniform disease that progresses in the same manner, and that tumor size might not be sufficient in defining stage and rate of progression. The degree of invasiveness of adenocarcinoma appears to play a role in predicting prognosis, and this is especially significant in small lung cancers. In parallel, advances in imaging techniques have allowed for better identification of the presence of lymphadenopathy and in the evaluation of heterogeneity in the composition of nodules on CT scan. This has led several researchers to try and search for a correlation between imaging findings and the pathological invasiveness of the SCLC. Special interest was given to radiological findings on HRCT scans of ground-glass appearance. Ground-glass appearance is defined as a homogeneous increase in density on $\mathrm{CT}$ scan without covering underlying vascular markings. Kodama et al were the first to study this correlation on 104 patients with small adenocarcinomas less than $2 \mathrm{~cm}$ in diameter between 1995 and 1999. Three independent radiologists semiquantitatively scored the extent of GGO on HRCT as greater than or less than $50 \%$. Three independent pathologists semiquantitatively scored the extent of the bronchioloalveolar carcinoma (BAC) component of the tumor on histologic examination as greater than or less than $50 \%$. A BAC area on pathology of less than
$50 \%$ was detected on HRCT as GGO of greater than $50 \%$ in only two patients (1.9\%). The sensitivity and specificity of GGO to diagnose BAC was $76 \%$ and $95 \%$, respectively. The 3 year-relapse-free survival rates in each group of 52 patients with GGO greater than and less than $50 \%$ were $100 \%$ and $72 \%$, respectively, after a median follow-up of 24 months. Univariate analysis indicated that both smaller GGO and BAC areas were significantly correlated with cancer relapse ( $P=0.005$ and $P=0.002)$. The multivariate analysis revealed an independent prognostic influence of the BAC area on relapse-free survival (relative risk 0.07, $P=0.015$ ). A multivariate analysis could not be performed in the group of GGO greater than $50 \%$, as until the end of the study date no relapses had been observed. ${ }^{15}$

The search for preoperative predictive indicators, such as GGO area, to predict the potential of relapse in patients with small adenocarcinomas arising in the peripheral lung continued, as this may be able to justify a smaller extent of resection, such as segmentectomy or even wedge resection, as being curative in intent and result. Shimada et al retrospectively analyzed the charts of 363 patients with cT1aN0M0 peripheral NSCLC for preoperative factors that may predict pathologically invasive tumor characteristics. These patients were suspected to have cT1aN0M0 peripheral NSCLC based on an HRCT scan of 1 or $2 \mathrm{~mm}$-slice intervals done within 1 month prior to surgical resection. The cases of cancer with invasive factors (CIF) were defined by the presence of vessel invasion, pleural invasion, or lymph-node metastasis on pathology. Clinical predictive factors studied were male sex and smoking status. Radiological predictive factors that were reviewed on HRCT were tumor-disappearance ratio (TDR), vascular convergence, air bronchograms, pleural indentation, and speculation. TDR was defined as follows: on an HRCT scan slice, the maximal tumor dimension was measured in a lung setting $\left(\mathrm{D}_{\mathrm{L}}\right)$; in a mediastinal setting, the ground-glass area disappears, leaving only the area of consolidation; the remaining maximal dimension of the consolidation area is measured $\left(\mathrm{D}_{\mathrm{M}}\right)$. TDR is calculated as $1-\mathrm{D}_{\mathrm{M}} / \mathrm{D}_{\mathrm{L}}$.

Of the 363 patients, 121 (33.3\%) had CIF and 242 (66.7\%) had non-CIF. Vessel invasion was identified in 97 patients (26.7\%), pleural invasion in 50 patients $(13.8 \%)$, and lymph-node metastases in 31 patients $(8.6 \%)$, respectively. The presence of such a percentage of invasive features clearly indicates that tumor size may not suffice for lung cancer staging. The proportion of patients that remained recurrence-free at 5 years was significantly higher in patients who had nonCIF (95\%) vs patients with CIF (64.5\%), with $P<0.001$. After multivariate regression analysis, TDR $<0.5$, the presence of 
spiculation, and the absence of an air bronchogram were shown to be statistically significant independent predictors of CIF. The most important predictor was a TDR $<0.5$, with a risk ratio for CIF of $33.139(P<0.001)$, followed by the absence of an air bronchogram, with a risk ratio of 2.825 $(P<0.001)$, and by the presence of spiculation, with a risk ratio of $2.315(P=0.014)$.

Patients with CIF have a poorer prognosis and are at higher risk of relapse, as demonstrated in this study. The proportion of patients who were relapse-free at 5 years with lymph -node metastasis was $24.9 \%$, whereas it was $61.7 \%$ for those with vessel invasion, and in those with pleural invasion it was $59.5 \%$. By reversing the predictive indicators, the goal was to attempt to predict the cases with non-CIF and who thus would be good candidates for limited resection. If a single factor were to be chosen, then TDR $\geq 0.5$ provided the best accuracy, with 77 of 78 patients (99\%) with TDR $\geq 0.5$ correctly predicted as non-CIF and one patient with recurrence within 5 years after surgical resection. Among the two-factor combinations, a TDR $\geq 0.5$ and the absence of spiculation provided the best accuracy, with 57 of 58 patients (98\%) correctly predicted as non-CIF, and all patients in this group remained recurrence-free for 5 years after surgery. The three-factor combination of a TDR $\geq 0.5$, absence of spiculation, and presence of air bronchograms was also accurate in predicting non-CIF, with 31 of 32 cases $(97 \%)$ correctly predicted as non-CIF, and there was no patient with recurrence within 5 years after surgery. ${ }^{16}$ Further studies with a larger population are required to validate any of these predictive models.

Several radiological criteria to predict preoperative pathological invasiveness of early-stage NSCLC were also tested in the first prospective multi-institutional study done: the Japan Clinical Oncology Group (JCOG) 0201. The study tested three criteria, derived from previous retrospective studies, which were consolidation:tumor $(\mathrm{C}: \mathrm{T})$ ratio on lung window less than 0.5 , indicating a tumor with a large GGO area, TDR, and visual estimation of the consolidation component in patients with clinical stage IA, ie, T1N0M0. The definition of TDR and the definition of pathologically invasive cases were similar to the previous study mentioned.

A total of 545 patients were included in the analysis. The specificity and sensitivity of the diagnosis for pathologically invasive cancer based on the $\mathrm{C}$ : $\mathrm{T}$ ratio from the lung window was $96.4 \%$ (161 of $167,95 \%$ CI 92.3\%-98.7\%) and $30.4 \%$ ( 115 of $378,95 \%$ CI $25.8 \%-35.3 \%$ ), respectively. Specificity and sensitivity for the diagnosis of pathologically invasive cancer based on the TDR from the mediastinal window were
$89.8 \%$ (150 of $167,95 \%$ CI $84.2 \%-94 \%)$ and $44.4 \%$ (168 of $378,95 \%$ CI $39.4 \%-49.6 \%$ ), respectively. Specificity and sensitivity for the diagnosis of pathologically invasive cancer based on the visual estimation of consolidation being higher than 0.5 were $93.4 \%$ (156 of $167,95 \%$ CI $88.5 \%-96.7 \%$ ) and $37 \%$ (140 of $378,95 \%$ CI $32.2 \%-42.1 \%$ ), respectively.

As a result, the highest specificity was that of the C:T ratio, but the lower 95\% CI limit for specificity did not exceed the prespecified threshold of $97 \%$. The highest specificity was obtained from the C:T ratio and the lowest from the TDR method. Conversely, the highest sensitivity was found with the TDR method, and the lowest from the C:T ratio. Therefore, if the TDR method were used to determine radiological early lung cancer, more invasive cancers would be misdiagnosed as radiologically noninvasive. This situation should be avoided as much as possible, because an invasive cancer would be resected using a limited resection, which is ill suited for such cancers. Conversely, the $\mathrm{C}$ : $\mathrm{T}$ ratio provided clinically safe criteria to identify noninvasive cancers. Analysis was repeated on the tumors that were $2 \mathrm{~cm}$ or less, with different cutoff values for $\mathrm{C}$ : $\mathrm{T}$ ratio of 0.25 and 0.75 . In the repeat analysis, the specificity of the diagnosis for pathologically invasive cancer was highest when the predictive factor was a $\mathrm{C}$ :T ratio from the lung window with a cutoff value of 0.25 , which was $98.7 \%$ (78 of $79,95 \%$ CI $93.2 \%-100 \%$ ). The lower limit of the $95 \% \mathrm{CI}$ is still however lower than the prespecified threshold of $97 \%$. Based on this analysis, the best candidates that ought to be selected are patients with tumor size less than $2 \mathrm{~cm}$ with a C:T ratio less than $0.25 .{ }^{17}$

A multidisciplinary approach to lung cancer by radiologists, surgeons, and pathologists thus proved to be a requirement for better management of lung adenocarcinoma. Based on this, the International Association for the Study of Lung Cancer (IASLC), American Thoracic Society (ATS), and European Respiratory Society (ERS) in 2011 suggested a new international multidisciplinary classification of lung adenocarcinoma based mainly on histology, but also taking into consideration clinical, molecular, radiological, and surgical issues that arise. One of the major modifications in the classification of small lung adenocarcinomas was that the terms "bronchoalveolar carcinoma" and "mixedsubtype adenocarcinoma" are no longer used. For resection specimens, new concepts were introduced for small solitary adenocarcinomas, such as adenocarcinoma in situ (AIS) in cases of pure lepidic growth and minimally invasive adenocarcinoma (MIA) in cases of predominant lepidic growth with $5 \mathrm{~mm}$ invasion. These histologies define patients who will have, if they undergo complete resection, $100 \%$ or 
near $100 \%$ disease-specific survival, respectively. Now that we further understand the pathological subtypes and their degree of invasiveness, it was suggested that the TNM staging would include in addition to tumor size an invasiveness component that was pathologically diagnosed or radiologically measured. ${ }^{18}$

Takahashi et al set out to confirm the prognostic significance, as well as to study the correlation, between radiological findings on preoperative HRCT and tumor invasiveness, as defined by the new IASLC/ATS/ERS classification, for pathologic stage IA lung adenocarcinoma. They studied three radiological parameters: GGO ratio, TDR, and consolidation diameter (CD). GGO ratio (\%) was defined as ( $1-$ [maximum dimension of consolidation on the lung window setting/ maximum dimension of the tumor in the lung window setting]) $\times 100$. TDR was defined similarly to previous studies mentioned, and CD was defined as the largest diameter of consolidation in the lung window setting in millimeters. Pathologic data were defined according to the new IASLC/ ATS/ERS classification.

They retrospectively reviewed 123 consecutive patients with pathologic stage IA lung adenocarcinoma, of which 54 had non-IAs. In this group, no recurrence was detected and 5-year OS rate was $100 \%$. Of note, 30 patients in this group had undergone lobectomy and 24 limited resection; 69 patients had IAs, with eight patients having tumor recurrences and a 5-year OS rate of 78.4\% (95\% CI 64.5\%-92.2\%). In this group, 53 patients had undergone lobectomy, while 16 patients had undergone limited resection. The difference in 5-year OS rates between the two groups was statistically significant, with $P<0.01$. Optimal cutoff values for GGO ratio, TDR, and CD were determined to be $50 \%, 75 \%$, and 10 $\mathrm{mm}$, respectively. The relationship between the radiological parameters and pathologic invasiveness of lung adenocarcinoma according to the cutoff values was investigated. With these cutoff values, GGO ratio, TDR, and CD showed positive predictive values of greater than $90 \%$ each. Clinically significant predictors of IA were the GGO ratio $(\leq 50 \%$ vs $>50 \%, P<0.01)$, TDR $(\leq 75 \%$ vs $>75 \%, P<0.01), \mathrm{CD}(\leq 10$ $\mathrm{mm}$ vs $>10 \mathrm{~mm}, P<0.01)$, tumor size ( $\leq 20 \mathrm{~mm}$ vs $>20 \mathrm{~mm}$, $P=0.048)$, and preoperative serum carcinoembryonic antigen level ( $\leq 5 \mathrm{ng} / \mathrm{mL}$ vs $>5 \mathrm{ng} / \mathrm{mL}, P<0.01)$. Interestingly, tumor size was the least significant predictive factor, with an OR of 2.3 vs GG ratio, which was the highest, with an OR of 65.3. This study's results thus validate the prognostic significance of the new classification. ${ }^{19}$

Diagnosing pathological invasiveness based on radiological findings thus appears to be a viable option, and further advancement in the field of radiology will allow for increased accuracy and increased predictive value. A different approach, after assessing for tumor size, would be to use intraoperative frozen sections (FS) taken during sublobar resection to decide on pathological invasiveness and subsequently convert to a complete lobectomy with mediastinal lymph-node dissection if needed in eligible patients with sufficient cardiopulmonary reserve.

At the time of the publication of the new IASLC/ATS/ ERS classification, it was judged that the role of intraoperative FS was not yet clearly defined. This is due to the fact that the predictive value of FS reported in the literature ranged from $93 \%$ to $100 \%$, but not all articles clearly reported the accuracy of FS analysis. ${ }^{16}$ Liu et al recently reviewed the records of 803 patients with clinical stage I peripheral lung adenocarcinoma who had undergone sublobar resection and in which FS was used to guide surgical strategy. The FS results were stratified into atypical adenomatous hyperplasia, AIS, MIA, and IA according to the IASLC/ATS/ERS lung adenocarcinoma classification. There were 125 cases of discrepancy between FS and final pathology, thus achieving a total concordance rate of FS of $84.4 \%$. When patients with atypical adenomatous hyperplasia, AIS, and MIA were classified together as a low-risk group, the concordance rate was $95.9 \%$. This is because most discrepancies $(n=92$, $73.6 \%$ ) were among atypical adenomatous hyperplasia, AIS, and MIA. If we add the six cases of the low-risk group that turned out to be benign on final pathology, then in 98 of the 125 errors of FS, the discrepancy did not affect the clinical outcome of patients, because their resection types were adequate. Of note, tumor size was a significant factor affecting the diagnostic accuracy of FS. FS diagnostic accuracy of for tumors $\leq 1 \mathrm{~cm}$ in diameter and larger than $1 \mathrm{~cm}$ was $79.6 \%$ and $90.8 \%$, respectively. A total of 301 patients were chosen for prognosis analysis. On follow-up, the 5-year recurrence free survival rate $(100 \%)$ was significantly better for patients with AIS/MIA than for patients with IA $(74.1 \%, P<0.01)$. However, the 5 -year OS rate $(100 \%)$ of patients with AIS/ MIA was not significantly different than for patients with IA $(88.3 \%, P>0.05) .{ }^{14}$

Two other studies analyzed the diagnostic accuracy of FS for pulmonary nodules based on the IASLC/ATS/ERS classification with conflicting results. Yeh et al studied FS and permanent section slides from 361 resected stage I lung adenocarcinomas $\leq 3 \mathrm{~cm}$ to test for agreement between FS and final diagnosis, as well as interobserver agreement, among five pathologists. Specifically, for agreement on degree of invasion, 35 cases were evaluated to determine the accuracy 
and interobserver agreement for discriminating between AIS, MIA, lepidic-predominant adenocarcinoma and IAs. There were only two cases of AIS, the interpretation was consistent with final pathology, and there was interobserver agreement; $79 \%$ of interpretations using FS were accurate for lepidicpredominant adenocarcinoma. In cases of MIA, however, only $41.3 \%$ were accurate for the diagnosis, and more than half $(52 \%)$ of interpretations were overdiagnosed as IA..$^{20}$ The sample size used was significantly smaller in this study, and thus the statistical power was lower, which could explain the differences in results. This is however consistent with a study done by Walts and Marchevsky on FS from 224 consecutive primary pulmonary adenocarcinomas, where only $59 \%$ of the FS diagnoses were correct for AIS and $46 \%$ for MIA. In comparison, $97 \%$ of the FS diagnoses were correct for IA. The most common FS error was overdiagnosis of MIA as IA. ${ }^{21}$

\section{Invasive and noninvasive approaches}

In addition to deciding on whether lobectomy or sublobar resection is the optimal choice for surgical treatment, an added option to consider is using either an open or a VATS approach. It is important to note that a large multi-institutional prospective randomized controlled trial comparing these two approaches is lacking, and all the data so far has been collected from retrospective data or from small randomized controlled trials. The data, however, are consistent, with multiple advantages for VATS lobectomy over open thoracotomy. In a meta-analysis done by Cao et al that included three studies with propensity-matched and -unmatched patients comparing VATS lobectomy to open thoracotomy, perioperative mortality was significantly lower for VATS compared to open thoracotomy in unmatched patients, but no significant difference was detected among propensity score-matched patients. Similar results were achieved for the complications that were studied, such as prolonged air leaks and sepsis. Overall, perioperative morbidity rate, incidence of pneumonia and atrial arrhythmias, and shorter duration of hospitalization were found to be significantly lower in patients undergoing VATS lobectomy compared to patients who underwent open thoracotomy, regardless of propensity matching. ${ }^{22}$

The main debate regarding oncological efficiency of the VATS approach stems from a lower rate of nodal upstaging described while using the VATS approach. Two big national registry-database queries have shown this association. A query of the Society of Thoracic Surgery database for clinical stage I NSCLC resected using lobectomy or segmentectomy between 2001 and 2010 showed a total of 11,531 resections; 7,137 of those resections were done using the open approach, and 4,394 the VATS approach. Nodal upstaging was seen in $14.3 \%(1,024)$ in the open group and $11.6 \%(508)$ in the VATS group $(P<0.001)$. Upstaging from N0 to N1 was more common in the open group $(9.3 \%$ versus $6.7 \%, P<0.001)$; however, upstaging from N0 to N2 was similar ( $5 \%$ open and $4.9 \%$ VATS, $P=0.52$ ). Among 2,745 propensity-matched pairs, N0-N1 upstaging remained less common with VATS (6.8\% versus $9 \%, P=0.002) .{ }^{23} \mathrm{~A}$ different query this time of the Danish Lung Cancer Registry from 2001 to 2007 showed 1,513 cases of clinical stage INSCLC. Nodal upstaging was significantly higher while using an open approach vs VATS approach for $\mathrm{N} 1$ upstaging $(13.1 \%$ vs $8.1 \%, P<0.001)$ and N2 upstaging $(11.5 \%$ vs $3.8 \%, P<0.001)$. Interestingly, however, this was not reflected by worsening survival. The unadjusted survival was actually higher using the VATS approach, but after adjustment by multivariate analysis the survival difference was no longer significant (HR 0.98, 95\% CI 0.8-1.22; $P=0.88$ ). The authors concluded that the difference in nodal upstaging might have been due to selection bias, though the reasons remained unclear. ${ }^{24}$

Similarly, several retrospective series showed that the VATS approach appears to have similar oncologic efficacy. For example, in a single-institution retrospective series, Berry et al evaluated OS of 1,087 cases of any-stage NSCLC treated with lobectomies, where 610 were performed using the VATS approach and 477 using open thoracotomy. The 5-year OS rate observed was better in the VATS group (57.5\% vs $43.1 \%$, $P<0.001)$. After propensity-score analysis controlling for age, sex, tumor size, and stage was performed with the cohort being now limited to 560 patients (311 using VATS and 249 open thoracotomy), the difference in 5-year survival was no longer significant $(54.7 \%$ vs $48 \%, P=0.3) .{ }^{25}$ The authors did however stress the fact that for an oncologic equivalence to be assured, the extent of lymph-node resection should be the same as when using the open approach. A lower risk of postoperative complications and morbidity with equivalent oncological efficacy has allowed VATS to gain ground as the surgical approach of choice. A recent study into the current practice patterns of VATS lobectomies showed that lobectomies performed in 2014 were $44 \%$ more likely to be done by VATS than in 2010 (OR 1.44, 95\% CI 1.28-1.62; $P<0.0001){ }^{26}$

In parallel to studies looking at lobectomy vs sublobar resection, several studies have also looked at comparing VATS lobectomy vs VATS sublobectomy. In a recent metaanalysis done by Liu et al that included eight studies and compared patients with stage I NSCLC who underwent VATS lobectomy vs VATS sublobectomy, it was shown that the OS was better in VATS sublobectomy, with a combined HR of 1.45 (95\% CI 1.11-1.9, $P=0.007)$. However, subgroup 
analysis showed that OS was comparable between patients undergoing VATS segmentectomy and VATS lobectomy, with an HR of 1.19 (95\% CI 0.67-2.1, $P=0.56)$. OS using VATS lobectomy was significantly higher than VATS wedge resection, with an HR of 4.19 (HR 4.19, 95\% CI 2.19-8.03; $P<0.0001)$. The evidence, however, was derived from small retrospective series with low evidence levels, so these conclusions are to be taken into consideration with a lot of reserve. ${ }^{27}$ VATS enables a different approach to what is basically the same procedure in terms of indications and contraindications. The only additional contraindication that is specific to the VATS approach is large tumor masses that are central in location. Previously, a history of thoracic surgeries or the presence of pleural adhesions were considered relative contraindications, but this is no longer valid. Inability to tolerate single-lung ventilation remains a potential contraindication for VATS. Clinically, absolute contraindications for open surgery can also be considered contraindications for VATS (eg, forced expiratory volume in 1 second $<30 \%$ and/or lung diffusion capacity for carbon monoxide $<30 \%$ ) yet for selected patients, such as the elderly with poor lung reserve, certain surgeons might consider VATS to be the only viable surgical option for them. ${ }^{28}$

A newly emerging technique is robotic surgery for lung cancer. Proponents claim additional benefits of improved ergonomics, three-dimensional optics, and $7^{\circ}$ EndoWrist capabilities, allowing more thorough lymph-node dissection and simplifying the operative procedure. A propensitymatched analysis comparing robotics and VATS to open lung resection of different stages was done by Bao et al, with 69 pairs selected after propensity matching. No statistically significant differences were seen regarding mean length of postoperative stay, chest-tube duration, number of lymph nodes retrieved, stations of lymph nodes resected, operative blood loss, or morbidity rates. However, robotics were associated with higher cost (US\$12,067 $\pm 1,610$ vs $\$ 8,328 \pm 1,004$, $P<0.001)$ and longer operative time $(136 \pm 40$ vs $111 \pm 28 \mathrm{~min}-$ utes, $P<0.001)$ than VATS. ${ }^{29}$ In the future, accumulation of surgeon experience, as well as a larger study, might be needed to demonstrate clinically significant advantages for robotics.

\section{Perioperative and long-term prognostic biomarkers currently being studied}

Even after complete resection of stage IA NSCLC, 5-year survival remains at $73 \%$, mainly attributed to local recurrence or progression of the disease. ${ }^{2}$ This helps in explaining lung cancer's position as the number-one cancer killer.
Several researchers have thus attempted to study prognostic biomarkers that would help us understand why surgical treatment of tumors that are similar according to current TNM staging yields different outcomes. In the evaluation of short term prognosis, B-type natriuretic peptide (BNP) has been studied, and was shown to be correlated with an increased rate of postoperative complications after earlystage NSCLC resection. In a study done by Nojiri et al on elderly patients undergoing pulmonary resection for lung cancer, a preoperative BNP level $>30 \mathrm{pg} / \mathrm{mL}$ had sensitivity of $79 \%$ and specificity of $83 \%$ for predicting postoperative cardiopulmonary complications after pulmonary resection for lung cancer. BNP was the most important predictor of postoperative complications in this study, of which the most common were cardiac arrhythmia, specifically atrial fibrillation, followed by pneumonia. ${ }^{30}$ Cagini et al also looked at the significance of the postoperative increase in BNP and whether this reflected a higher incidence of postoperative complications. They followed 294 patients undergoing noncardiac thoracic surgery with a median baseline BNP of $29.5 \mathrm{pg} / \mathrm{mL}$. They again demonstrated that patients who developed postoperative cardiopulmonary complications had at baseline significantly greater BNP values compared with those without complications ( 38.5 vs $26.5, P<0.0001$ ). Logistic regression analysis showed major pulmonary resections (lobectomies or pneumonectomies), BNP $\geq 118.5$ at postoperative day 1 , and age $\geq 65$ years to be the only independent predictive variables. In the subset of patients undergoing major pulmonary resection ( $\mathrm{n}=228$ ), $\mathrm{BNP} \geq 118.5 \mathrm{pg} / \mathrm{mL}$ at postoperative day 1 was associated with a 3.5 -fold increase in the risk of cardiopulmonary complications. ${ }^{31}$

As of the date of publication of this review, there has been no pathologic or serum prognostic marker adopted in the early-stage NSCLC population, while the ongoing research is vast. In the following paragraph, we list the different biomarkers currently under review. Diagnostic pathologic biomarkers for lung adenocarcinoma include CK7, TTF1, and napsin A. The prognostic value of these markers was studied by Ma et al. Patients with high expression levels of TTF 1 and napsin A, and high coexpression levels of TTF1-napsin A had better survival rates than those with low levels of expression. The expression levels of CK7 did not have any statistically significant effect on patients' survival..$^{32}$ Genome analyses of endothelial cells identified genes specifically expressed by tumor endothelial cells called tumor endothelial markers. In a retrospective study done by Pircher et al, correlation with clinical data revealed that increased tumor endothelial marker expression (Robo4, ECSCR, and Clec14) within NSCLC stromal tissue correlated with prolonged OS of 
operated NSCLC patients. ${ }^{33}$ MicroRNAs are small noncoding single-stranded RNAs approximately 19-22 nucleotides in length, which act by silencing mRNA, thus negatively regulating gene expression at the posttranscriptional level. Multiple key cell functions are under microRNA regulation, such as growth, proliferation, metabolism, and apoptosis. MicroRNAs have thus been studied in NSCLC for their diagnostic and prognostic value in both tissue diagnosis and more recently as circulating miRNAs. ${ }^{34}$ Pathologically, there have been several reports of the Let 7 miRNA family, as well as a study that looked at miR383 and its association with an improved clinical outcome in NSCLC. ${ }^{34,35}$ Elevated circulating miRNAs, such as miR486, miR195, miR30d, miR1, and miR499, were shown to be associated with improved survival. ${ }^{34,36}$ However, high miR125b, Let7f, and miR30e3p serum expression was reported to be an independent predictor of poor survival in NSCLC patients of various stages. ${ }^{34}$ EGFR status is a well-known predictive marker in stage IV NSCLC, yet its prognostic role in early-stage NSCLC is not well defined. Izar et al studied the prognostic role of EGFR status in treatment-naive resected stage I NSCLC, and found that mutation of the EGFR gene is a positive prognostic marker. ${ }^{37}$ Another predictive marker that has been well studied in advanced-stage NSCLC is the checkpoint inhibitor PDL1, yet its role in early-stage NSCLC is controversial. After studying two cohorts with a total of 544 patients, Velcheti et al found that the expression of PDL1 was a positive prognostic marker regardless of stage. ${ }^{38}$ More recently, however, Tsao et al studied the expression of PDL1 in 982 patients with resected NSCLC, and found that it was neither prognostic nor predictive of any benefit from adjuvant chemotherapy. ${ }^{39} \mathrm{Ki} 67$ is a nuclear nonhistone protein that is expressed during all phases of the cell cycle except the resting stage $\left(\mathrm{G}_{0}\right)$, and thus is a useful marker of proliferation. A meta-analysis of 32 studies involving 5,600 patients done by Wen et al showed a negative association between the level of Ki67 expression and OS and disease-free survival, especially in the subgroup of Asian patients with stage I-II adenocarcinoma. ${ }^{40} \mathrm{MIC} 1$, a member of the TGF $\beta$ superfamily, was found to be prognostic and suggestive of poor early outcomes using a cutoff of $>1,465 \mathrm{pg} / \mathrm{mL}$ with $72.2 \%$ sensitivity and $66.1 \%$ specificity. ${ }^{41}$ Another aspect tackled by Spaks et al was the level of CXCL4, which in their study was found to be reflective of tumor angiogenic activity and associated with a higher relapse rate, as well as worse OS and disease-free survival. ${ }^{42}$ It is important to note, however, that this was a single-institution experience of only 50 patients with early-stage NSCLC undergoing surgical resection. Mortalin, a member of the HSP70 family, which is involved in multiple cellular processes, was found to be prognostic in patients with early-stage NSCLC. Mortalin overexpression was significantly correlated with high histological grades, advanced stages, lymph-node metastases, and lower diseasefree survival and OS. ${ }^{43}$ GLDC was recently described as a critical enzyme of tumor-initiating cells. HIF $1 \alpha$ is the unique subunit that determines HIF-system activity, thereby regulating the adverse effects of hypoxia on cancer outcome. The combination of low-GLDC-negative HIF $1 \alpha$ expression was significantly prognostic for longer survival in resected early-stage NSCLC, as demonstrated by Berezowska et al. ${ }^{44}$ As the immune system plays a pivotal role in cancer, the study of immunorelated markers in the resected specimen may provide valuable prognostic information of NSCLC. The presence of $\mathrm{CD}^{+}$cells in the tumor compartment has been associated with better outcome, whereas the presence of FOXP3 $3^{+}$cells in the tumor-near stromal area was associated with worse OS. ${ }^{45}$

All of these prognostic factors studied could either serve as potential targets for future therapy or help delineate a treatment plan in the decision for adjuvant chemotherapy or more stringent criteria for surveillance in tumors with poor prognostic factors. To date, however, none of these prognostic factors has been validated in a Phase III clinical trial with regard to their effect on OS after treatment allocation based on their presence. Further studies are needed in this regard.

\section{Conclusion and ongoing trials}

The hope is that the answer to all the previous questions and controversies will be derived from two major multiinstitutional prospective randomized trials currently investigating the intentional use of sublobar resections for stage IA NSCLC. The first is under way in North America, Alliance/CALGB $140503,{ }^{46}$ and the other, which has completed enrollment is in Japan - JCOG0802/WJOG4607. ${ }^{47}$ The main difference between these two studies and the LCSG trial is that tumor size is now limited to less than or equal to $2 \mathrm{~cm}$ in both trials. The Alliance/CALGB 140503 trial has a target of 1,258 patients, and it includes both segmentectomy and wedge resection as an intentional treatment option compared with lobectomy. The primary end point studied is disease-free survival, and the secondary end points studied are OS, local and systemic recurrence, and pulmonary function, as measured by expiratory flow rate 6 months postoperatively. The JCOG0802/WJOG4607 trial has a target of 1,100 patients, and only intentional segmentectomy is used for comparison with lobectomy. The primary end point studied is OS, and the secondary end points are disease-free survival, local recurrence, and perioperative complications. 


\section{Disclosure}

The authors report no conflicts of interest in this work.

\section{References}

1. Siegel RL, Miller KD, Jemal A. Cancer statistics, 2016. CA Cancer J Clin. 2016;66(1):7-30.

2. Goldstraw P, Crowley J, Chansky K, et al. The IASLC Lung Cancer Staging Project: proposals for the revision of the TNM stage groupings in the forthcoming (seventh) edition of the TNM classification of malignant tumours. J Thorac Oncol. 2007;2(8):706-714.

3. National Comprehensive Cancer Network. Non-small cell lung cancer (version 3.2017). 2016. Available from: http://www.nccn.org/professionals/physician_gls/pdf/nscl.pdf. Accessed June 8, 2017.

4. Ginsberg RJ, Rubinstein LV. Randomized trial of lobectomy versus limited resection for T1 N0 non-small cell lung cancer. Ann Thorac Surg. 1995;60(3):615-623.

5. Moyer VA. Screening for lung cancer: U.S. Preventive Services Task Force recommendation statement. Ann Intern Med. 2014;160(5): $330-338$.

6. Landreneau RJ, Sugarbaker DJ, Mack MJ, et al. Wedge resection versus lobectomy for stage I (T1 N0 M0) non-small-cell lung cancer. J Thorac Cardiovasc Surg. 1997;113(4):691-700.

7. Khullar OV, Liu Y, Gillespie T, et al. Survival after sublobar resection versus lobectomy for clinical stage IA lung cancer. J Thorac Oncol. 2015;10(11):1625-1633

8. Cao C, Chandrakumar D, Gupta S, Yan TD, Tian DH. Could less be more? A systematic review and meta-analysis of sublobar resections versus lobectomy for non-small cell lung cancer according to patient selection. Lung Cancer. 2015;89(2):121-132.

9. Okada M, Nishio W, Sakamoto T, et al. Effect of tumor size on prognosis in patients with non-small cell lung cancer: the role of segmentectomy as a type of lesser resection. J Thorac Cardiovasc Surg. 2005;129(1):87-93.

10. Bao F, Ye P, Yang Y, et al. Segmentectomy or lobectomy for early stage lung cancer: a meta-analysis. Eur J Cardiothorac Surg. 2014;46(1):1-7.

11. Mery CM, Pappas AN, Bueno R, et al. Similar long-term survival of elderly patients with non-small cell lung cancer treated with lobectomy or wedge resection within the Surveillance, Epidemiology, and End Results database. Chest. 2005;128(1):237-245.

12. Razi SS, John MM, Sainathan S, Stavropoulos C. Sublobar resection is equivalent to lobectomy for T1a non-small cell lung cancer in the elderly: a Surveillance, Epidemiology, and End Results database analysis. J Surg Res. 2016;200(2):683-689.

13. El-Sherif A, Fernando HC, Santos R, et al. Margin and local recurrence after sublobar resection of non-small cell lung cancer. Ann Surg Oncol. 2007;14(8):2400-2405.

14. Liu S, Wang R, Zhang Y, et al. Precise diagnosis of intraoperative frozen section is an effective method to guide resection strategy for peripheral small-sized lung adenocarcinoma. J Clin Oncol. 2016;34(4):307-313.

15. Kodama K, Higashiyama M, Yokouchi H, et al. Prognostic value of ground-glass opacity found in small lung adenocarcinoma on highresolution CT scanning. Lung Cancer. 2001;33(1):17-25.

16. Shimada Y, Yoshida J, Hishida T, Nishimura M, Ishii G, Nagai K. Predictive factors of pathologically proven noninvasive tumor characteristics in T1aN0M0 peripheral non-small cell lung cancer. Chest. 2012;141(4):1003-1009.

17. Suzuki K, Koike T, Asakawa T, et al. A prospective radiological study of thin-section computed tomography to predict pathological noninvasiveness in peripheral clinical IA lung cancer (Japan Clinical Oncology Group 0201). J Thorac Oncol. 2011;6(4):751-756.

18. Travis WD, Brambilla E, Noguchi M, et al. International Association for the Study of Lung Cancer/American Thoracic Society/European Respiratory Society international multidisciplinary classification of lung adenocarcinoma. J Thorac Oncol. 2011;6(2):244-285.
19. Takahashi M, Shigematsu Y, Ohta M, Tokumasu H, Matsukura T, Hirai T. Tumor invasiveness as defined by the newly proposed IASLC/ATS/ ERS classification has prognostic significance for pathologic stage IA lung adenocarcinoma and can be predicted by radiologic parameters. J Thorac Cardiovasc Surg. 2014;147(1):54-59.

20. Yeh YC, Nitadori J, Kadota K, et al. Using frozen section to identify histologic patterns in stage I lung adenocarcinoma $\leq 3 \mathrm{~cm}$ : accuracy and interobserver agreement. Histopathology. 2015;66(7):922-938.

21. Walts AE, Marchevsky AM. Root cause analysis of problems in the frozen section diagnosis of in situ, minimally invasive, and invasive adenocarcinoma of the lung. Arch Pathol Lab Med. 2012;136(12):1515-1521.

22. Cao C, Manganas C, Ang SC, Yan TD. A meta-analysis of unmatched and matched patients comparing video-assisted thoracoscopic lobectomy and conventional open lobectomy. Ann Cardiothorac Surg. 2012;1(1):16-23.

23. Boffa DJ, Kosinski AS, Paul S, Mitchell JD, Onaitis M. Lymph node evaluation by open or video-assisted approaches in 11,500 anatomic lung cancer resections. Ann Thorac Surg. 2012;94(2):347-353.

24. Licht PB, Jørgensen OD, Ladegaard L, Jakobsen E. A national study of nodal upstaging after thoracoscopic versus open lobectomy for clinical stage I lung cancer. Ann Thorac Surg. 2013;96(3):943-950.

25. Berry MF, D’Amico TA, Onaitis MW, Kelsey CR. Thoracoscopic approach to lobectomy for lung cancer does not compromise oncologic efficacy. Ann Thorac Surg. 2014;98(1):197-202.

26. Blasberg JD, Seder CW, Leverson G, Shan Y, Maloney JD, Macke RA. Video-assisted thoracoscopic lobectomy for lung cancer: current practice patterns and predictors of adoption. Ann Thorac Surg. 2016;102(6):1854-1862.

27. Liu Q, Wang H, Zhou D, Deng X, Min J, Dai J. Comparison of clinical outcomes after thoracoscopic sublobectomy versus lobectomy for stage I nonsmall cell lung cancer: a meta-analysis. $J$ Cancer Res Ther. 2016;12(2):926-931.

28. Vannucci F, Gonzalez-Rivas D. Is VATS lobectomy standard of care for operable non-small cell lung cancer? Lung Cancer. 2016;100:114-119.

29. Bao F, Zhang C, Yang Y, He Z, Wang L, Hu J. Comparison of robotic and video-assisted thoracic surgery for lung cancer: a propensity-matched analysis. J Thorac Dis. 2016;8(7):1798-1803.

30. Nojiri T, Inoue M, Yamamoto K, et al. B-type natriuretic peptide as a predictor of postoperative cardiopulmonary complications in elderly patients undergoing pulmonary resection for lung cancer. Ann Thorac Surg. 2011;92(3):1051-1055.

31. Cagini L, Andolfi M, Leli C, et al. B-type natriuretic peptide following thoracic surgery: a predictor of postoperative cardiopulmonary complications. Eur J Cardiothorac Surg. 2014;46(5):e74-e80.

32. Ma Y, Fan M, Dai L, et al. The expression of TTF-1 and napsin A in early-stage lung adenocarcinoma correlates with the results of surgical treatment. Tumor Biol. 2015;36(10):8085-8092.

33. Pircher A, Fiegl M, Untergasser G, et al. Favorable prognosis of operable non-small cell lung cancer (NSCLC) patients harboring an increased expression of tumor endothelial markers (TEMs). Lung Cancer. 2013;81(2):252-258.

34. Matikas A, Syrigos KN, Agelaki S. Circulating biomarkers in nonsmall-cell lung cancer: current status and future challenges. Clin Lung Cancer. 2016;17(6):507-516.

35. Shang Y, Zang A, Li J, et al. MicroRNA-383 is a tumor suppressor and potential prognostic biomarker in human non-small cell lung caner. Biomed Pharmacother. 2016;83:1175-1181.

36. Su K, Zhang T, Wang Y, Hao G. Diagnostic and prognostic value of plasma microRNA-195 in patients with non-small cell lung cancer. World J Surg Oncol. 2016;14(1):224.

37. Izar B, Sequist L, Lee M, et al. The impact of EGFR mutation status on outcomes in patients with resected stage I non-small cell lung cancers. Ann Thorac Surg. 2013;96(3):962-968.

38. Velcheti V, Schalper KA, Carvajal DE, et al. Programmed death ligand-1 expression in non-small cell lung cancer. Lab Invest. 2014;94(1):107116. doi:10.1038/labinvest.2013.130. 
39. Tsao MS, Le Teuff G, Shepherd FA, et al. PD-L1 protein expression assessed by immunohistochemistry is neither prognostic nor predictive of benefit from adjuvant chemotherapy in resected non-small cell lung cancer. Ann Oncol. 2017;28(4):882-889.

40. Wen S, Zhou W, Li C, et al. Ki-67 as a prognostic marker in earlystage non-small cell lung cancer in Asian patients: a meta-analysis of published studies involving 32 studies. BMC Cancer. 2015;15:520.

41. Liu Y, Wang X, Wang T, et al. [Macrophage inhibitory cytokine-1 (MIC1) as a biomarker for diagnosis and prognosis of stage I-II non-small cell lung cancer]. Zhongguo Fei Ai Za Zhi. 2016;19(4):207-215. Chinese.

42. Spaks A, Svirina D, Spaka I, et al. CXC chemokine ligand 4 (CXCL4) is predictor of tumour angiogenic activity and prognostic biomarker in non-small cell lung cancer (NSCLC) patients undergoing surgical treatment. Biomarkers. 2016;21(5):474-478.

43. Sun J, Che SL, Piao JJ, Xu M, Chen LY, Lin ZH. Mortalin overexpression predicts poor prognosis in early stage of non-small cell lung cancer. Tumor Biol. 2017;39(3):1-9.
44. Berezowska S, Galván JA, Langer R, et al. Glycine decarboxylase and HIF-1 $\alpha$ expression are negative prognostic factors in primary resected early-stage non-small cell lung cancer. Virchows Arch. 2017;470(3): 323-330.

45. Usó M, Jantus-Lewintre E, Bremnes RM, et al. Analysis of the immune microenvironment in resected non-small cell lung cancer: the prognostic value of different T lymphocyte markers. Oncotarget. 2016;7(33):52849-52861

46. Alliance for Clinical Trials in Oncology. Comparison of different types of surgery in treating patients with stage IA non-small cell lung cancer. Available from: https://www.cancer.gov/about-cancer/treatment/clinical-trials/search/view?cdrid=555324. NLM identifier: NCT00499330. Accessed February 7, 2017.

47. Nakamura K, Saji H, Nakajima R, et al. A phase III randomized trial of lobectomy versus limited resection for small-sized peripheral nonsmall cell lung cancer (JCOG0802/WJOG4607L). Jpn J Clin Oncol. 2010;40(3):271-274.
Cancer Management and Research

\section{Publish your work in this journal}

Cancer Management and Research is an international, peer-reviewed open access journal focusing on cancer research and the optimal use of preventative and integrated treatment interventions to achieve improved outcomes, enhanced survival and quality of life for the cancer patient. The manuscript management system is completely online and includes
Dovepress

a very quick and fair peer-review system, which is all easy to use. Visit http://www.dovepress.com/testimonials.php to read real quotes from published authors. 